\title{
Polar-interferometry: What can be learnt from the IOTA/IONIC experiment
}

\author{
Jean-Baptiste Le Bouquin $^{a}$, Karine Rousselet-Perraut ${ }^{b}$, Jean-Philippe Berger ${ }^{b}$, Emilie \\ Herwats $^{b}$, Myriam Benisty $^{b}$, Olivier Absil ${ }^{b}$, Denis Defrere ${ }^{c}$, John Monnier ${ }^{d}$, Wesley Traub $^{e}$ \\ ${ }^{a}$ European Southern Observatory, Casilla 19001, Santiago 19, Chile \\ ${ }^{b}$ Lab. d'AstrOphysique de Grenoble, France \\ ${ }^{c}$ Univ. de Liege, Belgium \\ ${ }^{d}$ Department of Astronomy, University of Michigan, Ann Arbor, MI., USA \\ $e$ Jet Propulsion Lab., USA
}

\begin{abstract}
We report the first near-IR polar-interferometric observations, performed at the IOTA array using its integrated optics combiner IONIC. Fringes have been obtained on calibration stars and resolved late-type giants. Optical modeling of the array and dedicated laboratory measures allowed us to confirm the good accuracy obtained on the calibrated polarized visibilities and closure phases. However, no evidences for polarimetric features at high angular resolution have been detected. The simulations and the results presented here open several perspectives for polar-interferometry, especially in the context of fibered, single-mode combiners.
\end{abstract}

Keywords: Optical Long Baseline Interferometry ; Polar-Interferometry ; IOTA ; IONIC

\section{INTRODUCTION}

One of the main technical hurdle for optical long baseline interferometry (LBI) is the control of polarization. Moreover, analyzing the polarimetric content of the interferometric signal can bring important, new, polarimetric constraints on the observed target at high angular resolution. In this context, several theoretical studies have pointed out the interests of developing polarimetric analyzer for LBI, ${ }^{1,2}$ mainly for stellar astrophysics. So far, very few experience on sky have been reported, and all of them have been done at visible wavelengths. ${ }^{3-5}$

Apart from the technical challenge, the main difficulty is the amplitude of the polar-interferometric signal, generally of the order of few percents or even less at the near-infrared wavelengths (when talking about scattering induced polarization). Detecting such a signal with decent confidence requires a significantly better accuracy on the raw interferometric data obtained in individual polarization states. In the near-infrared, single-mode filtering by the mean of fibers and integrated optics has already proved to be the key to access sub-percent precision interferometry. Yet, the possibility of doing polar-interferometry with fibers has generally been dropped out because of the high intrinsic polarization effects of such devices. This is however only partially true: the birefringence between 2 perpendicular axes is indeed high, but the polarization cross-talk between these axes can stay below $1 \%$, assuming polarization-maintaining fibers are properly used. So, even if circular-polarization analysis is compromised by the unknown phase shift between mechanically fixed linear axes, linear polarization analysis along these axes may still be possible. Demonstrating it is the purpose of this study.

Before it closed in 2006, the IOTA array combined 3 telescopes in the near-IR by the mean of a fibered integrated optics beam combiner called IONIC. ${ }^{6}$ Since the beginning, provision has been taken to put a movable Wollaston prism between the combiner output and the detector, to separately image both vertical and horizontal linearly-polarized states. In this paper, we investigate the capability of such a facility to perform and calibrate linear polar-interferometric observations. We first recall the main instrumental systems of the IOTA array (Sec. 2). We describe the measurement done across the IOTA optical chain to characterize its polarimetric behavior, with a special emphasis on the beam combiner itself (Sec. 3). We then build a simple numerical model to estimate the impact of the instrumental polarization in the measured visibilities in natural and polarized

Send correspondence to J.B Le Bouquin: E-mail: jlebouqu@eso.org, Telephone: +56 24633087

Optical and Infrared Interferometry, edited by Markus Schöller, William C. Danchi, Françoise Delplancke Proc. of SPIE Vol. 7013, 70130F, (2008) · 0277-786X/08/\$18 - doi: 10.1117/12.786377 

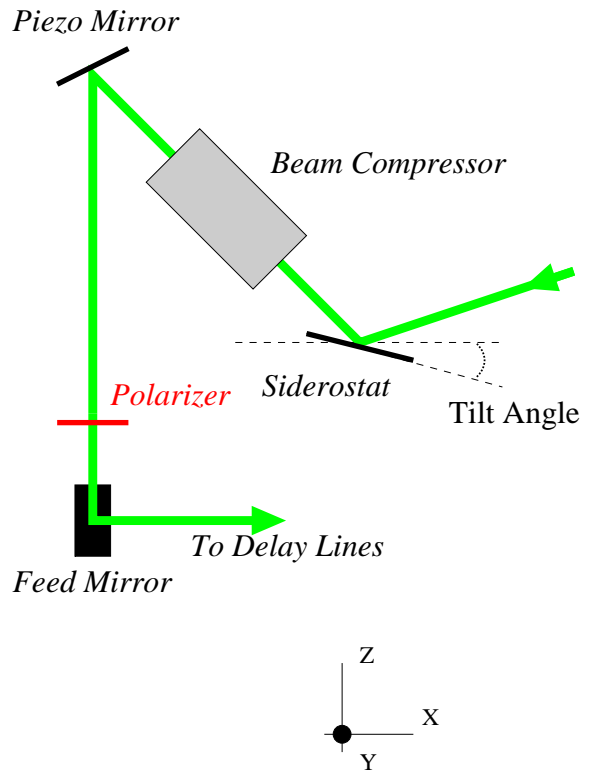

A)
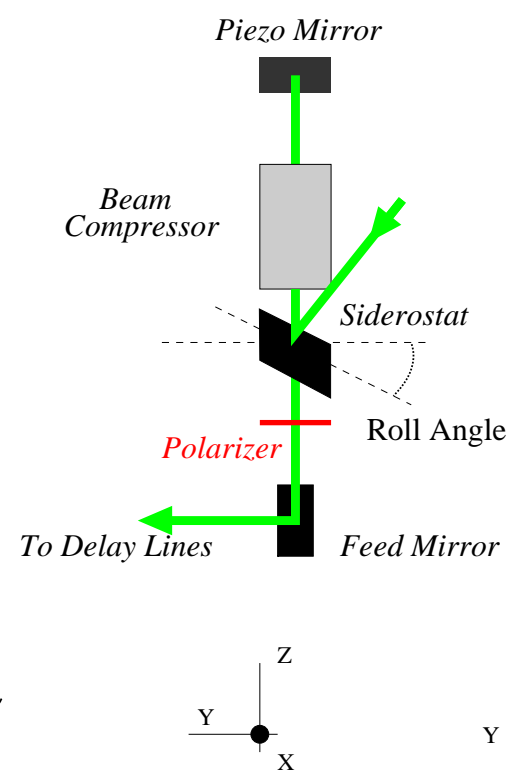

B)
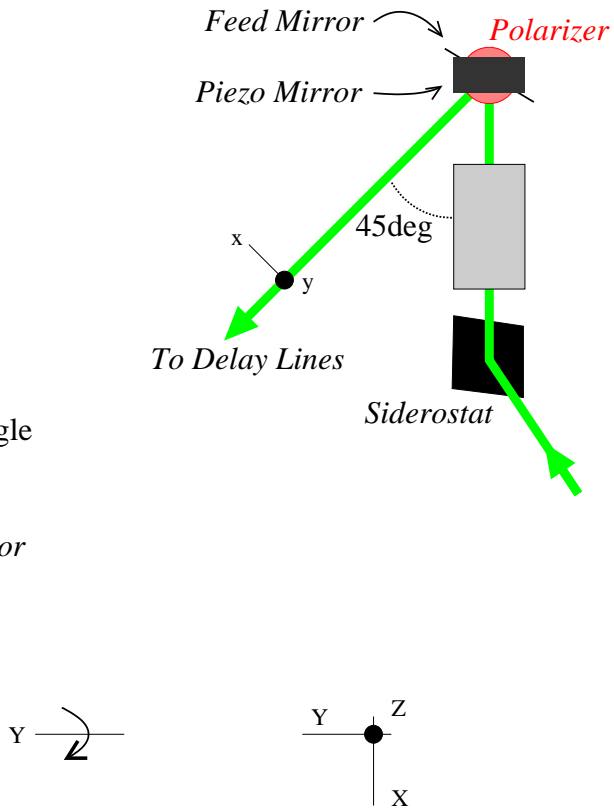

C)

Figure 1. Sketch of the telescopes optical design with three different projection views. The $Z$ direction points to the zenith. The star is pointed and tacked with the Siderostat mirror through the Tilt and Roll angles (see view A and B). The Siderostat mirror rotates the stellar beam from the Roll Angle $(r)$ when feeding the beam compressor (see view B). The beam compressor (a pair of convex mirrors with quasi-normal incidence) has only very marginally effect on the polarization. The Feed mirror rotates the telescope beam from $45^{\circ}$ when feeding the Delay Lines (see view $\mathbf{C}$ ). The red slide denotes the position where we put the linearly polarized light source when we measured extinction curves from the telescope.

light (Sec. 4). We present an application of this work on an attempt to detect near-IR polar-interferometric signal due to dust-scattering around two evolved late-type giants (Sec. 5). Finally, the paper ends with our recommendations for building facilities allowing linear polar-interferometric observations (Sec. 6).

\section{THE IOTA INSTRUMENT}

The Infrared Optical Telescope Array $\left(\right.$ IOTA* $^{*}$ was a three arms interferometer located at Mt. Hopkins, Arizona (US). Main systems were: 3 relocatable siderostats serving as flux collectors, 3 fix, long delay lines allowing to roughly equalize the optical path of each arm, 2 short delay lines used to perform the fine, real-time optical path equalization, a visible tip-tilt sensor in the laboratory actuating tip-tilt mirrors located in the siderostats, and several beam-combiners. To maximize the interferometric efficiency, its followed the so-called golden rule, ${ }^{7}$ meaning that all optical arms encountered the same series of optical reflections, from the telescopes to the interferometric beam combiner. For the need of this paper, we focus the description on two interesting parts only: the flux collectors and the 3-beam integrated beam-combiner, because they concentrate the large majority of polarimetric effects of the facility.

As showed in Fig. 1, each flux collector is composed of a motorized siderostat used to point and track the observed star, a beam-compressor, a piezo driven tip-tilt mirror redirecting the beam to toward the ground, and a feed mirror to finally redirect it horizontally toward the Delay Lines. Important to notice, the vertical plane defined by the feed mirror, the piezo mirror and the siderostat mirror $(\{X, Z\}$ plane in Fig. 1$)$ is $45^{\circ}$ rotated compare to the plane defined by the feed mirror, the piezo mirror, and the output beam feeding the Delay Lines. After the reflexion on the feed mirror, the beam is propagated through the Delay Lines up to the beam combiner without any additional rotation.

\footnotetext{
*http://tdc-www.harvard.edu/IOTA/
} 


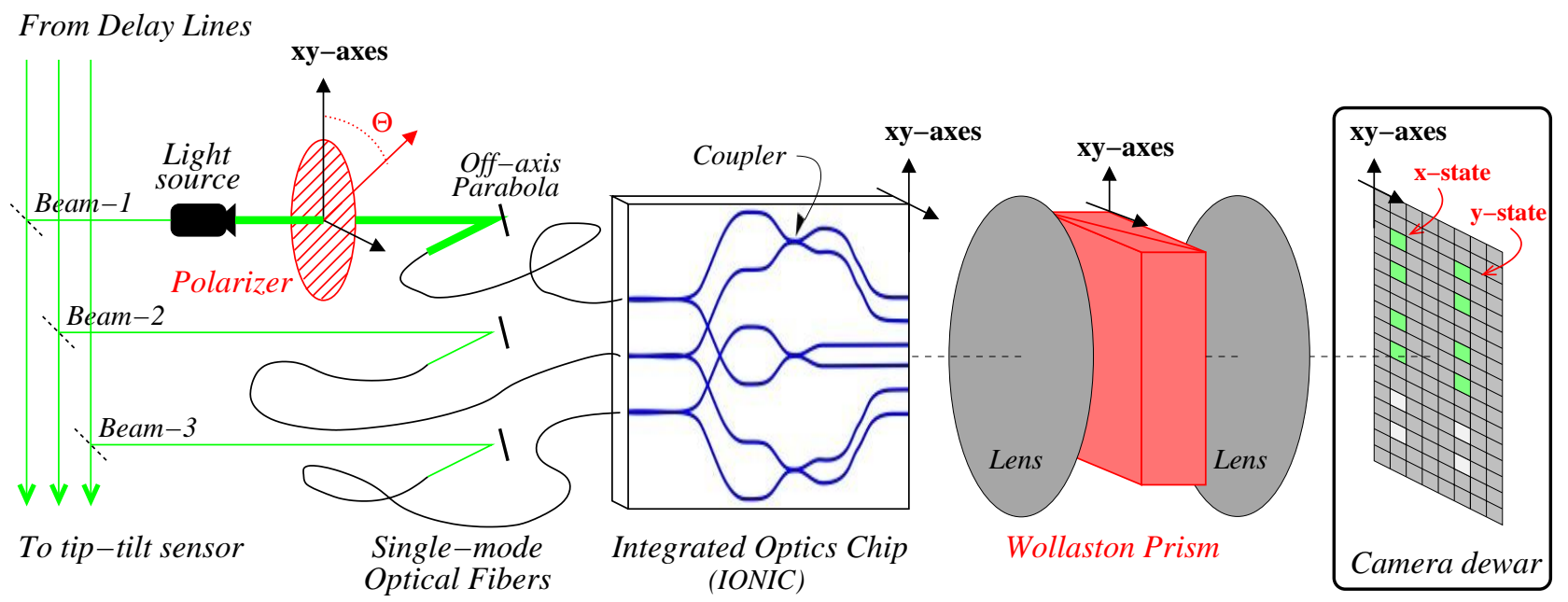

Figure 2. Sketch of the IOTA laboratory when using the IONIC beam combiner. The $\{x, y\}$ reference frame correspond to the vertical and horizontal directions inside the laboratory. In this example, the light source is placed just before the injection optics of beam-1, leading to the outputs $\{1,2,3,4\}$ to be illuminated.

After being equalized in optical delay by the Long and Short Delay Lines, the beams enter the focal laboratory (see Fig. 2). While visible part of the light feed the tip-tilt sensor, dichroics deflect the infrared part toward the IONIC bench. Off-axis parabola feed single-mode polarization-maintenance fibers, glued to the integrated optics chip. The latter performs a pairwise beam combination by the mean of 3 Y-junctions and 3 directional couplers. ${ }^{6}$ The light coming out of those six outputs is then focused onto 6 pixels of the infrared camera. A Wollaston prism, or a simple dispersive prism, can be inserted between the chip and the detector in order to separately images two polarization states or several spectral channels. The chip itself uses silica on silicon technology, and results from a collaboration between LAOG and CEA-LETI. It was generally operated in the astronomical $H$-band (1.5-1.8 microns). Concerning the design, no photometric calibration signals were used since the information on photometric coupling fluctuations can be retrieved from algebraic considerations on the interferometric signals.

\section{LABORATORY MEASUREMENTS}

The aim of these studies was to measure the cross-talk effects between the two perpendicular directions of polarization throughout the IOTA optical train. The basic principle of such a measurement lies in (i) totally polarizing the input light along a direction $x$ at the entrance of the train and (ii) analyzing the polarization of the output light at various levels of the optical train. If the optical train does not introduce polarization, the flux recorded is maximal along the direction $x$ and null along the direction perpendicular to $x$. If a non-null flux is measured along the latter, this flux is called the cross-talk level.

\subsection{Instrumental setups}

We used the following experimental setup to measure the polarimetric behavior of the interferometer:

- Polarizer: a light source followed by a polarizer on a rotation mount was used to create a fully linearly polarized beam with defined orientation. Even if the precision of the mount itself is much better, the accuracy of positioning the polarizer inside the mount is of the order of $0.5 \mathrm{deg}$.

- Analyzer: a Wollaston prism was inserted between the integrated optic combiner and the detector, in a collimated beam (see Fig. 2). This allows to separate and image simultaneously the horizontal and vertical linear polarizations on the detector. A proper alignment between the Wollaston axes and the polarimetric axes of the integrated chip is mandatory to avoid polarization cross-talk. By design, the chip outputs are physically aligned with its mechanical axes, that are the facets. Due to the constraints during its manufacturing, the combiner has polarimetric axes that correspond to its own symmetry axes, i.e. the axis 

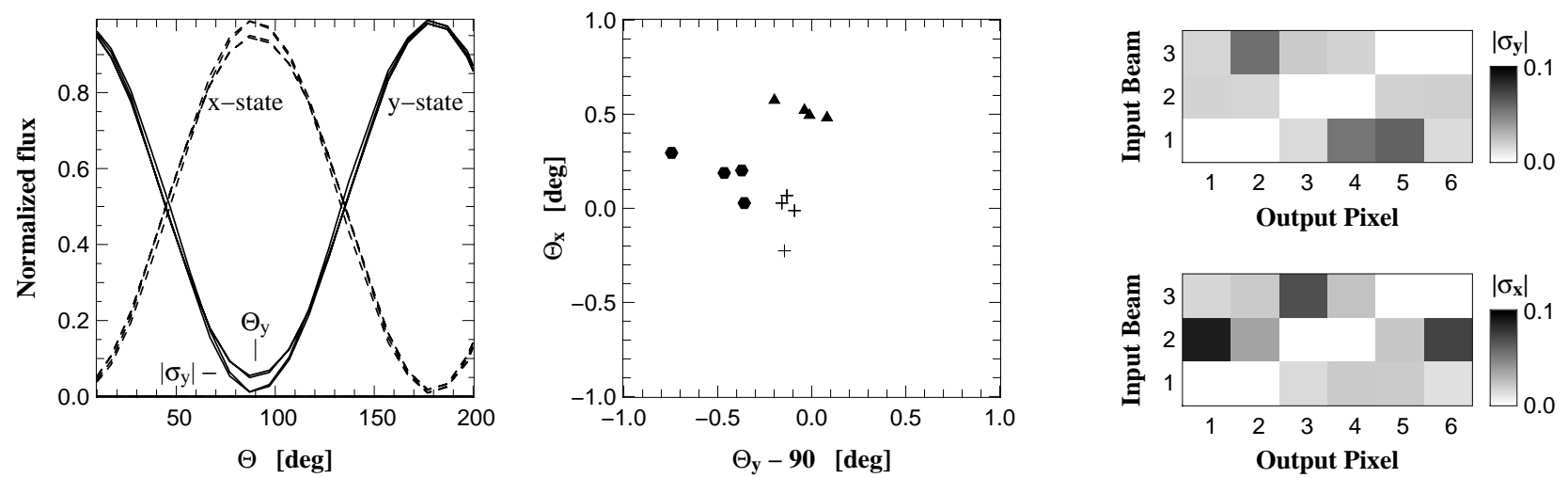

Figure 3. Left: Example of extinction curves obtained with setup described in Sec. 3.1, here with internal light placed in beam A before the combiner. Middle: measured neutral-axis $\Theta$ (i.e angle of each minimum) for each polarization and for the 3 different input beams (symbols). Right: measured cross-talk $|\sigma|$ (i.e normalized flux value of each minimum), for the 6 -output pixels by the 2 -input beams by the 2 -polarizations.

normal to the chip plane and the axis in the plane chip that is perpendicular to the propagation direction. Alignment of the Wollaston prism with respect to the detector is ensured by a correct horizontal centering of the output spots on the detector and a vertical alignment of the spots between the two polarizations. This vertical/horizontal frame is called $x y$ reference in the following (see Fig. 2).

\subsection{Data acquisition and reduction}

To obtain extinction curves, we measure the flux level on the 12 output spots ( 6 combiner outputs in 2 polarization states) versus the input polarization angle. We repeat the measure for each input beam. We normalize by the total amount of flux in the 12 output spots. This removes all photometric effects coming from source polarization and/or injection quality. Typical extinction curves are displayed in left panel of Fig. 3, for the November 2005 campaign. We then fit each minimum and determine its exact value (hereafter called cross-talk amplitude, $|\sigma|$ ) and angle (hereafter called neutral-axis, $\Theta$ ).

The experiment has been repeated three times: (i) in November 2005, with the internal light source just before the off-axis parabola to investigate the polarization behavior of the beam-combiner alone ; (ii) in November 2005 , with the light source placed on the so-called pipe (i.e. as close as possible of the siderostat) to investigate the polarization behavior of the IOTA optical train; and (iii) in March 2006, with the light source before the parabola. The exact positions of the light source in each of the experience are emphasized in red in Fig. 1 and 2.

\subsection{Results from laboratory measurements}

Middle panel of Fig. 3 plots the measured neutral-axis $\Theta$. All neutral-axis are well-aligned, whatever the input beam and the output pixel considered. The observed dispersion is below our precision $\left( \pm 1^{\circ}\right)$. This proves that first the fiber heads are all well oriented in both input and output (glued with the integrated optic combiner). Secondly, the chip itself does not rotate the polarization and is properly aligned with the Wollaston. Important to notice, the neutral-axis of both polarizations are separated by $90^{\circ}$ exactly.

Right panel of Fig. 3 plots the cross-talk, that is the residual flux level $|\sigma|$ obtained at angle $\Theta$, for the 6 -output pixels by the 2-input beams by the 2-polarizations. In ideal conditions, all these values should be zero. Note that the white diagonal is meaningless since it corresponds to non-existing input-beam/output-pixel pairs. Clearly the 3 internal couplers of the integrated combiner always have 2 bad cross-talk values over the 8 measured. This can be explained by the integrated optics manufacturing process: several silica layers of various indices are deposited on a silicon substrate and etched to create channel waveguides. The etching is not complete and a thin guiding sub-layer that can guide light remains. Strikingly, the 3 couplers show 3 different behaviors. It is therefore not easy to explain the cross-talk with a simple physical effect inside the chip. 

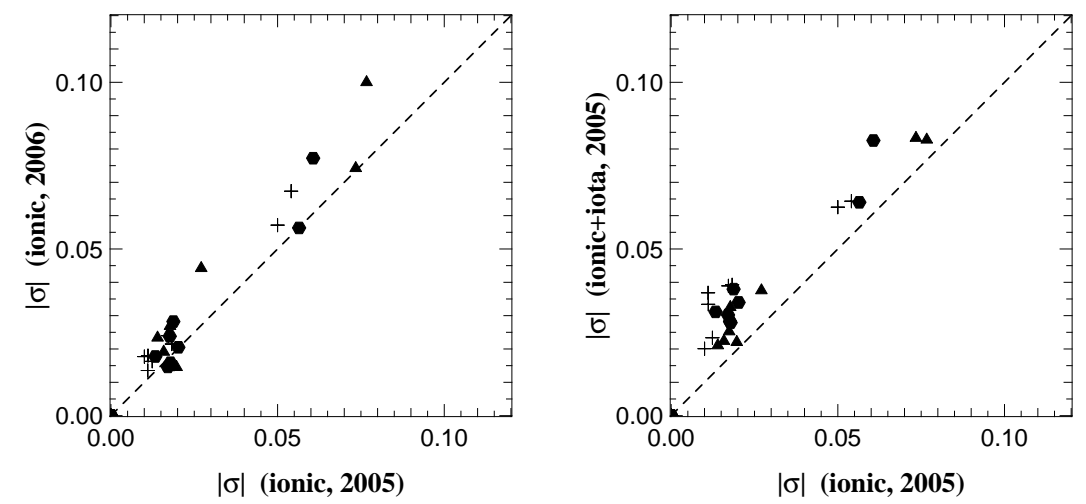

Figure 4. Left: cross-talk values with internal light placed before the combiner measured in 2006 versus same quantities measured in 2005. Right: cross-talk values with internal light placed at the telescope versus the same quantities but with internal light placed before the combiner. Dashed line is the $x=y$ relation.

The measures of cross-talk are repeatable from November 2005 to March 2006, even if a marginal increase of $\times 1.1$ can perhaps be noticed (Fig. 4, left). Additionally, the small shift of $1 \%$ when measuring the cross-talk from the telescopes (Fig. 4, right) is possibly explained by little optical misalignments in the IOTA optics. Yet, the cross-talk is maintained below $10 \%$, with the majority of the measures below $4 \%$.

We were not able to measure simply the relative transmission between the two neutral-axis, mainly because the fibers injection quality was obviously dependent on the polarizer angle. Yet, when the input polarizer is at $45^{\circ}$, the output flux ratio gives a rough estimation of the relative transmission between the linear axes. We estimate the relative transmission from the telescopes (not included) to the detector to be

$$
\left|\tau_{y}\right| \sim 0.9
$$

which is compatible with typical flux ratio obtained on sky (where the target is supposed to be unpolarized and well-injected).

\section{NUMERICAL SIMULATIONS}

Based on our laboratory measurements, we build a numerical model of the IONIC+IOTA instrument, in order to: (i) study the effect of polarization in natural light fringes, especially in the context of high-accuracy calibration; and (ii) test the capability of such facility to perform and calibrate polar-interferometric observations.

\subsection{Mathematical formalism}

We use the polarized routines developed for our simulation code VITRUVsim. ${ }^{8}$ This codes implements the formalism developed for radio-astronomy by Hamaker et al., ${ }^{9}$ in which the coherency vector is defined as:

$$
\mathbf{E}_{\mathrm{AB}}=<\mathbf{e}_{\mathrm{A}} \otimes \mathbf{e}_{\mathrm{B}}{ }^{*}>=\left(\begin{array}{c}
<e_{\mathrm{A} x} e_{\mathrm{B} x}{ }^{*}> \\
<e_{\mathrm{A} x} e_{\mathrm{B} y}{ }^{*}> \\
<e_{\mathrm{A} y} e_{\mathrm{B} x}{ }^{*}> \\
<e_{\mathrm{A} y} e_{\mathrm{B} y}{ }^{*}>
\end{array}\right)=\left(\begin{array}{c}
E_{\mathrm{AB} x x} \\
E_{\mathrm{AB} x y} \\
E_{\mathrm{AB} y x} \\
E_{\mathrm{AB} y y}
\end{array}\right)
$$

where $\mathrm{A}$ and $\mathrm{B}$ are the optical beams considered, and $e_{x}$ and $e_{y}$ are the polarization components of the electric field in the $x y$ coordinate frame. The coherency vector is linked to the polarized image of the observed target with the generalized Zernick and Van Cittert theorem:

$$
\mathbf{E}_{\mathrm{AB}}=\int\left(\begin{array}{ccc}
I(\vec{\alpha}) & + & Q(\vec{\alpha}) \\
U(\vec{\alpha}) & +i & V(\vec{\alpha}) \\
U(\vec{\alpha}) & -i & V(\vec{\alpha}) \\
I(\vec{\alpha}) & - & Q(\vec{\alpha})
\end{array}\right) \exp \left(2 i \pi \vec{\alpha} \overrightarrow{\mathcal{B}}_{\mathrm{AB}} / \lambda\right) \mathrm{d} \vec{\alpha}
$$



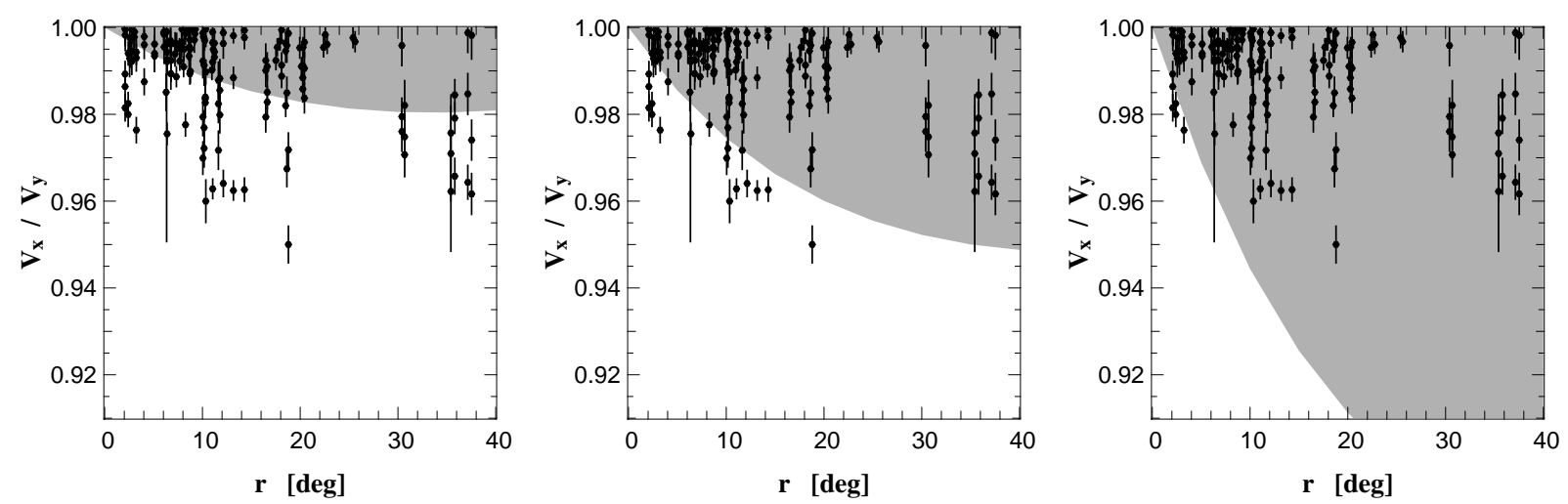

Figure 5. Observed ratio between polarized transfer-function versus the $r$ angle of siderostat mirror (points), overlaid by result of simulation (shaded region). The simulation result is not to a line but to a region because we computed a grid of scenario corresponding to (i) different configuration of unknown instrumental phases (see Sec. 4.2) and (ii) different target polarization (circular, linear, resolved, unresolved) and degree of polarization (ranging from unpolarized to $10 \%$ polarized). Simulation has been done with different values of $\left|\tau_{\text {sider }}\right| \sim\left|\tau_{\text {piezo }}\right|$, that is respectively $0.9,0.8$ and 0.6 for the left, middle and right panel. We qualitatively estimate that $\left|\tau_{\text {sider }}\right| \sim\left|\tau_{\text {piezo }}\right| \sim 0.8$ best reproduces the observations and we adopt this value for rest of the study.

where $\lambda$ is the effective wavelength of observation, $\overrightarrow{\mathcal{B}}_{\mathrm{AB}}$ is the interferometric baseline, that is the distance between the telescopes projected onto the sky, and $I(\vec{\alpha}), Q(\vec{\alpha}), U(\vec{\alpha}), V(\vec{\alpha})$ are the target images in the four Stokes components. Following Hamaker et al., ${ }^{9}$ coherency vectors are propagated through the instrument with the formula:

$$
\mathbf{E}_{\mathrm{AB}, \text { out }}=\left(\mathbf{J}_{\mathrm{A}} \otimes \mathbf{J}_{\mathrm{B}}\right) \mathbf{E}_{\mathrm{AB}, \text { in }}
$$

where $\mathbf{J}_{\mathrm{A}}$ and $\mathbf{J}_{\mathrm{B}}$ are the Jones matrix for beam $\mathrm{A}$ and $\mathrm{B}$ respectively. Yet, the coherency vector itself is not the observable in optical interferometry, but the visibilities. Visibilities in natural $\left(V_{\text {nat }}\right)$ and polarized lights $\left(V_{x}\right.$, $\left.V_{y}\right)$ can be written:

$$
V_{\mathrm{nat}}=\frac{\left|E_{\mathrm{AB} x x}+E_{\mathrm{AB} y y}\right|}{\sqrt{\left(E_{\mathrm{AA} x x}+E_{\mathrm{AA} y y}\right) \cdot\left(E_{\mathrm{BB} x x}+E_{\mathrm{BB} y y}\right)}} \quad ; \quad V_{x}=\frac{\left|E_{\mathrm{AB} x x}\right|}{\sqrt{E_{\mathrm{AA} x x} \cdot E_{\mathrm{BB} x x}}} \quad ; \quad V_{y}=\frac{\left|E_{\mathrm{AB} y y}\right|}{\sqrt{E_{\mathrm{AA} y y} \cdot E_{\mathrm{BB} y y}}}
$$

Note that visibilities are defined as ratio between mutual-coherency fluxes over self-coherency fluxes. Visibilities can be indifferently computed with the input coherency vectors (real visibilities of the target), or with the output coherency vectors (observed visibilities on the detector).

\subsection{Modeling the optical chain}

The full optical chain from the detector to the star (left to right) can be described by a Jones matrix of the form (one for each beam):

$$
\mathbf{J}(r, i)=\underbrace{\left(\begin{array}{cc}
1 & \sigma_{x} \\
\sigma_{y} & \tau_{y}
\end{array}\right) \times \mathcal{R}(\Theta)}_{\text {IONIC } \rightarrow \text { telescope }} \times \underbrace{\mathcal{R}\left(45^{\circ}\right) \times\left(\begin{array}{cc}
1 & 0 \\
0 & \tau_{\text {piezo }}
\end{array}\right)}_{\text {piezo mirror }} \times \underbrace{\mathcal{R}(r) \times\left(\begin{array}{cc}
1 & 0 \\
0 & \tau_{\text {sider }}(i)
\end{array}\right)}_{\text {siderostat mirror }}
$$

The left-most matrix describes the optics from the detector to the telescope (not-included). The non-diagonal terms are the cross-talk terms measured and discussed in previous section. The rotation matrix $\mathcal{R}(\Theta)$ denotes the orientation of the neutral-axis of the beam in the common $x y$ reference frame attached to the detector. Yet, this rotation is practically negligible, as seen in previous section. This part of the optical chain does not depends on pointing (except the delay-line position, which has very few influence on the polarization). $\tau_{\text {piezo }}$ is the complex differential transmissions due to the reflexion on the piezo mirror, which is rotated from $\mathcal{R}\left(45^{\circ}\right)$ compare to the laboratory (fixed by design, see Sec. 2 and Fig. 1). $\tau_{\text {sider }}$ is the complex differential transmissions due to the reflexion on the siderostat mirror. Its amplitude and phase depend on the incidence angle on the siderostat $(i)$. 

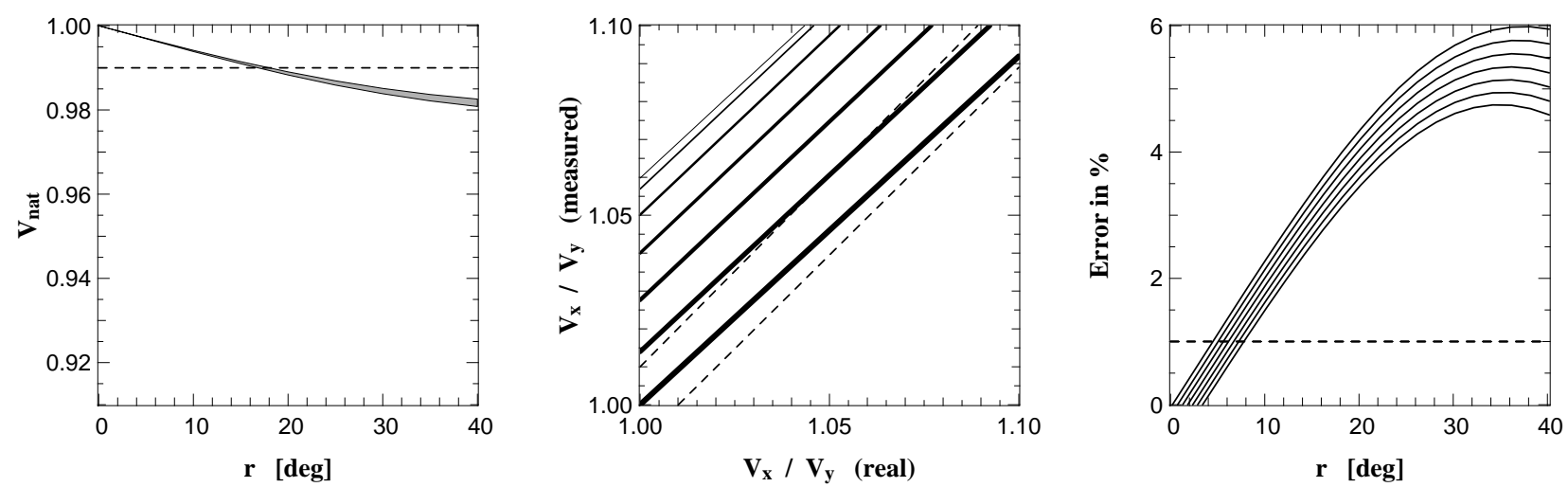

Figure 6. Left: Simulated transfer-function in natural light versus the $r$ angle of the siderostat mirror. The shaded region correspond to simulation of various target polarization (linear, circular, resolved, unresolved) and degree of polarization (ranging from unpolarized to $10 \%$ polarized). Middle: Measured polarimetric signal versus real polarimetric signal, for various angle $r$ (thick to thin lines ranging from 0 to $50 \mathrm{deg}$ ). Right: Error on the polarimetric signal versus the angle $r$, for different science target polarizations ranging from unpolarized (upper) to $10 \%$ polarized (lower). In all cases, results have been calibrated by an unresolved, unpolarized calibrator at $r=0$ deg. Dashed lines shows the $1 \%$ error limits.

Finally, the rotation matrix $\mathcal{R}(r)$ comes from the angle between the piezo mirror and the siderostat mirror (see Fig. 1). For information, $r$ and $i$ are the so-called roll and tilt angles in the IOTA nomenclature.

We started a numerical model of IOTA by filling the Jones matrix with all information about neutral-axis, transmission and polarization cross-talk values derived in the previous section. We add the following constraints: (i) $\arg \left\{\tau_{y}\right\}<<180 \mathrm{deg}$, otherwise the fringes in natural light would cancel and (ii) $\arg \left\{\sigma_{x}\right\} \sim \arg \left\{\sigma_{y}\right\} \sim 90 \mathrm{deg}$ because the measured neutral-axes are well orthogonal. Yet, several critical quantities are missing:

- To overcome the issue of remaining unknown phases $\left(\arg \left\{\tau_{y}\right\}, \arg \left\{\tau_{\text {piezo }}\right\}, \arg \left\{\tau_{\text {sider }}\right\} \ldots\right)$, we adopt a conservative strategy by computing a grid of phase configurations exploring the parameter space (about 5000 points) for each simulated scenario, and select the worst result. Therefore our simulations give upper limits on the polarimetric effect of the optical chain.

- We were not able to characterize the polarization amplitudes of the telescope itself (dominated by optical reflexions on the piezo and the siderostat mirrors). As shown in Fig. 5, we estimated the worst possible value of $\left|\tau_{\text {piezo }}\right|$ and $\left|\tau_{\text {sider }}\right|$ to be about 0.8 by comparing observations and simulations. We acknowledge that amplitude and phase of $\tau_{\text {sider }}$ are formally function of the siderostat inclination $i$, generally ranging from $10^{\circ}$ to $50^{\circ}$. It was not possible to really constraint this dependency. We decided to keep the previous upper-limit value in all following simulations.

\subsection{Results from numerical simulations}

Based on our simple modeling, we can infer some properties of the instrumental response in natural light (Fig. 6, left). First the transfer-function is natural light is only marginally dependents on the target polarization. We obtained a fluctuation of about $0.5 \%$ with a target polarization ranging from 0 to $5 \%$. On the other side, the transfer-function is slightly dependent on the $r$ angle. We predict an error of $1 \%$ when the siderostat mirror rotates from about $20 \mathrm{deg}$ (upper limit). Interestingly, this is fully compatible with the high-precision visibilities routinely obtained with the IOTA interferometer.

The second goal of our modeling was to test the capability of the facility to perform and calibrate polarinterferometric observations. To do so, we consider the interesting scientific signal to be the ratio of the visibilities obtained in linearly polarized lights $V_{x} / V_{y}$, coding for instance a difference of diameter. This ratio is calibrated by the observation of an unresolved, unpolarized target at $r=0$ deg. As seen in middle and right plots of Fig. 6, the siderostat rotation mainly introduces a bias on the measured signal. For the observer, this mimics a (or cancels any) polarimetric signal of the same level. Therefore claim of detection (or non detection) should 

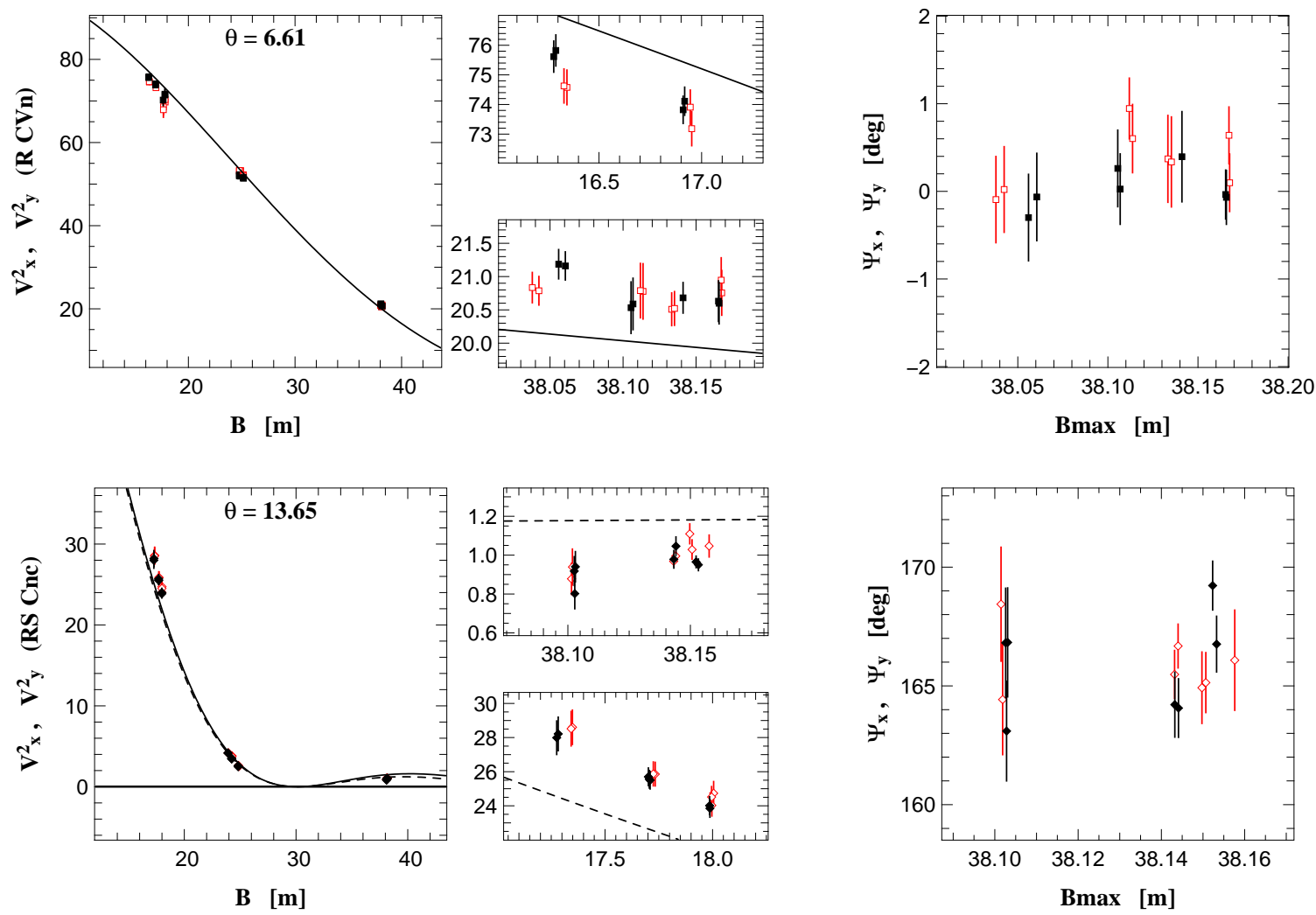

Figure 7. H-band polar-interferometric observations of R CVn (top) and RS Cnc (bottom) obtained in March 2006. Calibrated polarized visibilities are plotted on the 3 left panels, while calibrated polarized closure-phases are plotted on the right panel. Open red symbols and closed black symbols are for polarization states $x$ and $y$ respectively. Solid and dashed lines are best fit with Uniform Disk and Limb Darkened Disk models.

carefully take into account this potential effect. Quantitatively, this bias stays within $1 \%$ as long as the $r$ is smaller than $10 \mathrm{deg}$, but rapidly increase to several percents for largest angles. On the other side, and very interestingly, the bias amplitude is only slightly dependent on the polarization of the observed target.

\section{POLAR-INTERFEROMETRIC OBSERVATIONS OF LATE-TYPE GIANTS}

The photosphere of evolved M-giants is generally surrounded by molecular and dust shells due to their important mass loss. The net polarization arising from diffusion on such spherically symmetric shell is zero. Yet, with linear polar-interferometry, it becomes possible to separate it from the un-polarized photosphere. ${ }^{4}$ Although diffusion is less important in the near-infrared, it may be detected thanks to the high accuracy provided by single-mode filtering.

In March 2006, we perform polar-interferometric observations of the Mira variable R CVn and the SemiRegular pulsating RS Cnc. At this time, the detector didn't allow to record both polarization states simultaneously. We had to quickly switch from one to the other every $10 \mathrm{~min}$. Observations of calibrators have been interlaced every 20min. To keep the polarimetric bias as small as possible, we selected calibrators within $10 \mathrm{deg}$ of the science target (see Tab. 1). We then calibrate independently the transfer-function of both polarization states. Resulting visibilities and closure-phases are displayed in Fig. 7.

R CVn appears marginally resolved, with a best-fit Uniform Disk diameter of $\oslash_{\mathrm{UD}}=6.61 \mathrm{mas}$ (to be compared with 6.63mas as reported in CHARM2). On the other side, RS Cnc is fully resolved with a best-fit diameter of $\oslash_{\mathrm{UD}}=13.65 \mathrm{mas}$. Departure from model in the second visibility-lobe as well as non-null closure phases $\Psi$ 

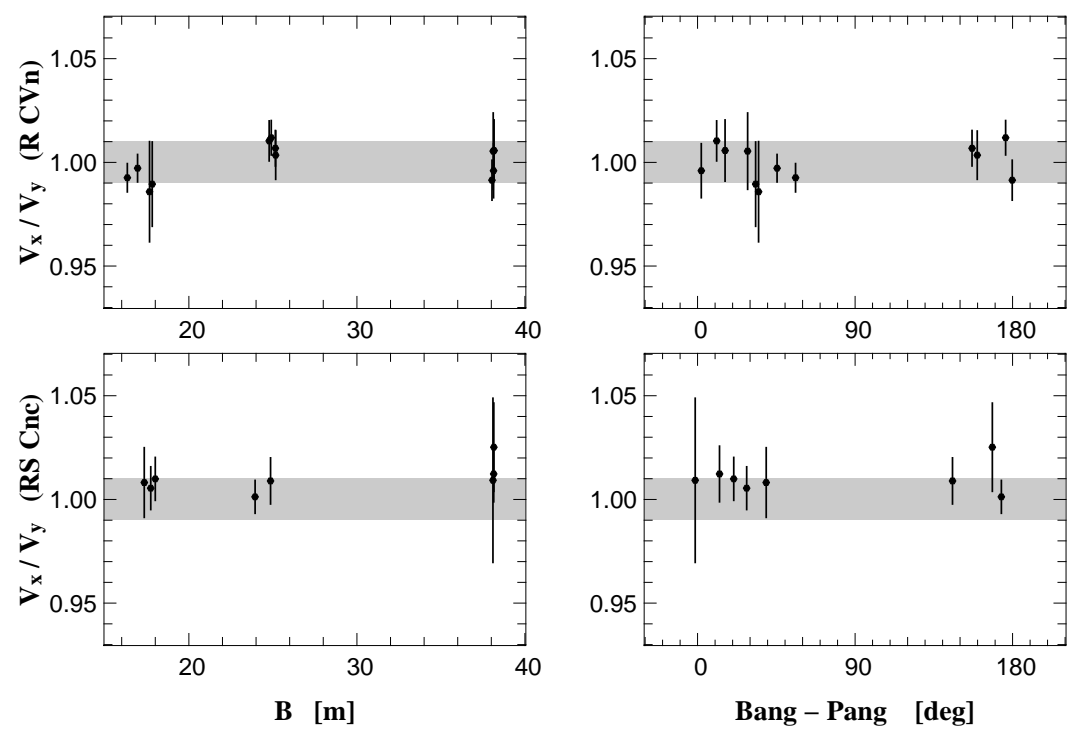

Figure 8. Calibrated ratio of polarized visibilities for R CVn (top) and RS Cnc (bottom) versus the baseline length (left) and versus the angle between baseline and analyzer as projected on sky (right). Shaded region is for $V_{x} / V_{y}=1.0 \pm 1 \%$, denoting the maximum potential bias level that we estimated on the previous section.

point on resolved features (large surface features, binary...). Yet, on both targets, no systematic differences are noticeable between the two polarization states. To better emphasizes the potential high angular resolution polarimetric signal, we display the ratio of polarized visibilities $\left(V_{x} / V_{y}\right)$ in Fig. 8. The overall accuracy on this quantity is in the range $\pm 0.5 \%$ to $\pm 2 \%$. On RS Cnc, data point appear systematically shifted toward $V_{x} / V_{y}=1.01$, but this falls into the possible bias amplitude computed in Sec. 4.3 (similar study can be done for the closure-phase). Therefore our conclusion is a non-detection of $H$-band polar-interferometric signal, at the level of $1 \%$ in visibilities and about $1 \mathrm{deg}$ in closure-phase. For comparison, the $V$-band polar-interferometric signal previously detected on Mira stars was of the order of $10 \%$ in visibilities (work of Ireland et al. with SUSI ${ }^{4}$ ).

\section{WHAT CAN BE LEARNT FROM THIS EXPERIMENT}

\subsection{Conclusions concerning the IOTA facility}

Concerning the IOTA array, several additional tests would have improve our understanding of its polarimetric behaviors: (i) The piezo mirror could have been better constrained with cross-talk measurements made with a polarizer inside the telescope itself (and not at its output, as done in our study). (ii) Part of the missing phase informations could have been measured by using circularly-polarized light. In addition of polarizer and Wollaston prism, such tests require quarter-wave plates at both extremities of the tested optical chain. (iii) Part of the missing phases and amplitudes could have been constrained by polar-interferometric observations with a polarizer in the telescope, the star just serving as spatially coherent source between the apertures.

Table 1. Observed targets with associated calibrators. Spectral types have been extracted from the Simbad database.

\begin{tabular}{|c|l|l|}
\hline Target Name & Spectral Type & Distance \\
\hline \hline R CVn & M6IIIe, Mira & $0 \mathrm{deg}$ \\
\hline HD116475 & M4III & $8.8 \mathrm{deg}$ \\
\hline HD121647 & K2III & $1.5 \mathrm{deg}$ \\
\hline
\end{tabular}

\begin{tabular}{|c|l|l|}
\hline Target Name & Spectral Type & Distance \\
\hline \hline RS Cnc & M6IIIase, SR & $0 \mathrm{deg}$ \\
\hline HD86513 & G9III & $10.6 \mathrm{deg}$ \\
\hline HD76219 & G8Iab & $4.5 \mathrm{deg}$ \\
\hline
\end{tabular}


Concerning the beam combination, the use of fibers and integrated optics does not prevent from linear polarization analysis, at least along the geometrical axes defined by the components. The fibered combiner has well aligned and stable neutral axis, but has relatively important polarization cross-talk, up to $8 \%$ for some outputs. Important to notice, the 3 internal couplers of the integrated combiner show 3 different polarization behaviors. It is therefore not easy to associate the cross-talk to a simple, physical effect inside the chip. However new technological processes allow to avoid a guiding sub-layer and thus to reduce the cross-talk effects.

Quite surprisingly, numerical simulations proved that the internal cross-talk does not destroy the capability of performing precise interferometry. Yet, to reach a sub-percent accuracy, one should take care of using a calibration star closer to the target than about $10^{\circ}$ (which is a lower limit, the exact value depending on the real polarimetric properties of the IOTA mirrors). This is to avoid any damageable effect of the siderostat rotation on the transfer-function.

\subsection{Golden-rule for linear polar-interferometry}

We conclude this paper by summarizing our expertise into a golden rule to build linear polar-interferometers. The classical golden rule avoids interferometric efficiency degradation because of polarization : the wavefront must experience reflections with the same sequence of direction and incidence angles between the point where the beams are divided (the telescopes) and the point where they are combined (the combiner). ${ }^{7}$ Yet, this rule only ensures that the polarization cross-talk and delay are similar on both arms, but does not prevent from an high cross-talk value. Even if not relevant when doing natural light interferometry, such polarimetric behavior is particularly complicated to calibrate when doing polar-interferometry, especially when dealing with birefringent optics such as highly tilted mirrors, fibers or integrated optics. When considering linear polar-interferometry, the golden rule should be revised into: the neutral-axes of all birefringent optical elements should be aligned with the axes along which the polarization is analyzed:

- All mirror axes should be aligned in the optical train from the telescope up to the focal laboratory. Note that the interferometric arms should not necessarily be symmetric (even if this is required if the facility is also used for interferometry in natural light).

- These axes should be aligned with the polarization axes of the combiner and of the analyzer. In case of an integrated optics beam combiner, the analyzer can be placed after the chip since the latter introduces few cross-talk as demonstrated in this paper.

- Telescope or siderostat mounts should have all mirror axes aligned. It is obviously impossible to reach such situation for all pointing position into the sky. However, with certain care, one can ensure that this is respected when pointing along a given plane in sky. This plane should imperatively be aligned with the optical train, to avoid any beam rotation that would create cross-talk. We note that the IOTA siderostats, that were $45 \mathrm{deg}$ rotated compare to the Delay Lines, are an example of the worst case!

- Targets will be preferably observed when crossing this plane, where linear polarization cross-talk is minimum. To cover a large declination range and to better observe at maximum elevation, one may prefer roughly align it with the North-South direction. The array should provide at least one baseline aligned with this axis, and possibly another one perpendicular to it. This would align the analyzer axes with the baselines, and maximize the efficiency to unveil symmetrically symmetric polarized structures.

We note that this modified golden rule is simultaneously more and less strident that the normal one: more strident because it forbids several concepts for building the telescopes (classical altitude-azimuth or equatorial mounts do not respect it, while Labery's "boule" telescope or simple siderostat do) ; less strident because the interferometric arms should not necessarily be identical. 


\section{REFERENCES}

[1] Rousselet-Perraut, K., Hill, L., Lasselin-Waultier, G., Boit, J.-L., Rousset, G., Blanc, J.-C., Moreaux, G., Corneloup, J. M., List, S., and Voet, C., "Field rotator for the GI2T interferometer," Optical Engineering 37, 610-615 (Feb. 1998).

[2] Elias, N. M., "Optical Interferometric Polarimetry. II. Theory," Astrophysical Journal 611, 1175-1199 (Aug. 2004).

[3] Brown, R. H., Davis, J., and Allen, L. R., "An attempt to detect a corona around beta ORI with an intensity interferometer using linearly polarized light.," Mon. Not. of the Royal Astron. Soc. 168, 93-100 (July 1974).

[4] Ireland, M. J., Tuthill, P. G., Davis, J., and Tango, W., "Dust scattering in the Miras R Car and RR Sco resolved by optical interferometric polarimetry," Mon. Not. of the Royal Astron. Soc. 361, 337-344 (July 2005).

[5] Rousselet-Perraut, K., Le Bouquin, J. B., Mourard, D., Vakili, F., Chesneau, O., Bonneau, D., Chevassut, J. L., Crocherie, A., Glentzlin, A., Jankov, S., Ménardi, S., Petrov, R., and Stehlé, C., "First sky validation of an optical polarimetric interferometer," Astron. \& Astrophys. 451, 1133-1137 (June 2006).

[6] Berger, J., Haguenauer, P., Kern, P. Y., Rousselet-Perraut, K., Malbet, F., Gluck, S., Lagny, L., SchanenDuport, I., Laurent, E., Delboulbe, A., Tatulli, E., Traub, W. A., Carleton, N., Millan-Gabet, R., Monnier, J. D., and Pedretti, E., "An integrated-optics 3-way beam combiner for IOTA," in [Interferometry for Optical Astronomy II. Edited by Wesley A. Traub. Proc. of SPIE, Vol. 4838, pp. 1099-1106.], 1099-1106 (Feb. 2003).

[7] Traub, W. A., "Polarization effects in stellar interferometers.," in [European Southern Observatory Astrophysics Symposia], European Southern Observatory Astrophysics Symposia 29, 1029-1038 (1988).

[8] Le Bouquin, J.-B., Herwats, E., Carvalho, M.-I., Garcia, P., Berger, J.-P., and Absil, O., "A numerical simulator for VITRUV," in [The power of optical/IR interferometry: recent scientific results and 2nd generation VLTI instrumentation, Proc. of the Conference], (2005).

[9] Hamaker, J. P., Bregman, J. D., and Sault, R. J., "Understanding radio polarimetry. I. Mathematical foundations.," Astronomy and Astrophysics, Supplement 117, 137-147 (May 1996). 